\title{
Study on Testing Methods for the Heat Transfer Performance of Plate Heat Exchangers
}

\author{
Bin Ren ${ }^{1,2^{*}}$, Xuchen $Z u^{1}$, Yannan $\mathrm{Du}^{1}$, Zhe $\mathrm{Pu}^{1}$, Hongliang $\mathrm{Lu}^{1,2}$, Aini $\mathrm{He}^{1,2}$ \\ ${ }^{1}$ Shanghai Institute of Special Equipment Inspection and Technical Research, Putuo District, Shanghai, 200062, China \\ ${ }^{2}$ National Heat Exchanger Product Quality Inspection Center, Jinshan District, Shanghai, 201518, China
}

\begin{abstract}
Plate heat exchangers are new-type compact heat exchangers with high heat transfer efficiency widely used in heating, food, medicine, shipbuilding and petrochemical industries. However, only the laboratory testing can accurately obtain the real heat transfer and flow resistance performance of plate heat exchanger. In this paper, the basic principles of modified Wilson plot method and equal velocity method are firstly introduced. Then the testing system including flow chart and testing instruments are discussed. Finally, contrast experiments using the different two methods are conducted. The results showed that for plate heat exchangers with equal channel, the equal velocity method and modified Wilson plot method can both be used to test the convective heat transfer performance of plate heat exchanger. The equal velocity method is recommended because the deformation of plate is relatively smaller.
\end{abstract}

\section{Introduction}

Plate heat exchanger is a kind of heat exchanger which is made of corrugated metal heat exchange plates stacking with each other. The cold fluid and hot fluid respectively flows through the channels formed by the two plates. The heat transfers through the middle plate. It is a new type of efficient compact heat exchanger, mainly used in heating, food, medicine, shipbuilding and petrochemical industries [1]. The core component of plate heat exchanger is the plate, which is rolled from a thin metal plate through a special mould. The shape, angle and depth of corrugation on the plate determine the heat transfer and flow resistance performance of the whole heat exchanger, which is the focus of engineering research.

Generally, it is difficult to obtain the real heat transfer and flow resistance characteristics of plate heat exchangers with new type plate by calculation or simulation [2]. As non-standard products, even using mature plates, it is also difficult to obtain the real performance due to the differences in process combinations. In addition, due to the existence of fouling resistance, the performance decreases as the operation time increases. Therefore, only the laboratory testing can accurately obtain the real heat transfer and flow resistance performance of plate heat exchanger.

The measurement of flow resistance performance is relatively simple. So, only the heat transfer performance testing method is introduced in this paper. The basic principles of modified Wilson plot method and equal velocity method are firstly introduced. Then the testing system including flow chart and testing instruments are discussed. Finally, contrast experiments using the different two methods are conducted.

\section{Testing method and system}

\subsection{Testing method}

The overall heat transfer coefficient can be calculated by measuring the flow rate of cold and hot fluids and the inlet and outlet temperature. The heat transfer correlation of cold and hot fluids further needs to separate from the overall heat transfer coefficient, so as to facilitate the design and selection of heat exchangers. The function form of the single-phase convective heat transfer correlation is generally known, and the undetermined coefficients can be obtained by modified Wilson plot method and equal velocity method. These two methods are also adopted by GB/T 27698-2011 [3].

When the fouling thermal resistance can be ignored, the total thermal resistance $R_{o v}$ of the whole heat transfer process can be divided into the cold side thermal resistance $R_{c}$, the wall thermal resistance $R_{w}$ and the hot side thermal resistance $R_{h}$. The formula is as following:

$$
R_{o v}=R_{c}+R_{w}+R_{h}
$$

The thermal resistances of cold and hot sides are respectively described as:

$$
\begin{aligned}
& R_{c}=\frac{1}{\alpha_{c}}=\frac{1}{\left(\lambda_{c} / d_{c}\right) \cdot N u_{c}}=\frac{1}{\left(\lambda_{c} / d_{c}\right) \cdot\left(C_{c} \cdot \operatorname{Re}_{c}^{P_{c}} \cdot \operatorname{Pr}_{c}^{0.4}\right)} \\
& R_{h}=\frac{1}{\alpha_{h}}=\frac{1}{\left(\lambda_{h} / d_{h}\right) \cdot N u_{h}}=\frac{1}{\left(\lambda_{h} / d_{h}\right) \cdot\left(C_{h} \cdot \operatorname{Re}_{h}^{P_{h}} \cdot \operatorname{Pr}_{h}^{0.3}\right)}
\end{aligned}
$$




$$
R_{w}=\frac{\delta}{\lambda_{w}}
$$

Considering that the total thermal resistance is the reciprocal of the overall heat transfer coefficient, substituting Eq. (2), Eq. (3) and Eq. (4) into Eq. (1), Eq. (1) can be transformed into:

$$
\frac{1}{K}=\frac{1}{\left(\lambda_{c} / d_{c}\right) \cdot\left(C_{c} \cdot \operatorname{Re}_{c}^{P_{c}} \cdot P r_{c}^{0.4}\right)}+\frac{\delta}{\lambda_{w}}+\frac{1}{\left(\lambda_{h} / d_{h}\right) \cdot\left(C_{h} \cdot \operatorname{Re}_{h}^{P_{h}} \cdot P r_{h}^{0.3}\right)}
$$

There are four undetermined coefficients $C_{c}, C_{h}, P_{c}$ and $P_{h}$ in Eq. (5). When the cold fluid channel is the same as the hot fluid channel and the cold working medium is the same as the hot working medium, $C_{c}$ is equal to $C_{h}$, and $P_{c}$ is equal to $P_{h}$. Therefore, only two undetermined coefficients need to be acquired. At this time, equal velocity method is used to obtain the convective heat transfer correlation of the plate heat exchanger. During the experiment, the flow velocity of cold fluid is adjusted to be the same as that of hot fluid. Then Eq. (5) can be rewritten into:

$C \cdot u^{P}=\frac{1}{1 / K-\delta / \lambda_{w}} \cdot\left[\frac{1}{\left.\left(\lambda_{c} / d\right) \cdot\left(d / v_{c}\right)^{P} \cdot P r_{c}^{0.4}\right)}+\frac{1}{\left.\left(\lambda_{h} / d\right) \cdot\left(d / v_{h}\right)^{P} \cdot P r_{h}^{0.3}\right)}\right]$

A linear equation can be obtained by taking logarithm on both sides of Eq. (5). The values of $C$ and $P$ can be calculated by least square method $[4,5]$.

When the cold fluid channel is different with the hot fluid channel or the cold working medium is different with the hot working medium, the thermal resistance of the fluid on one channel must remain constant (here it is assumed that the thermal resistance of hot fluid is constant). Then modified Wilson plot method can be used to acquire the convective heat transfer correlation and Eq. (5) can be rewritten into [6]:

$$
C_{c} \cdot \operatorname{Re}_{c}^{P_{c}}=\frac{1}{(1 / K-b) \cdot\left(\lambda_{c} / d_{c}\right) \cdot P r_{c}^{0.4}}
$$

\subsection{Testing system}

The testing system for convection heat transfer is shown in Fig. 1. Vapour is served as the heating source and air is served as the cooling source. The hot medium is heated by vapour before entering the testing sample. Then the hot medium transfers heat to the cold medium in the testing sample. After leaving the testing sample, the cold medium is finally cooled by air, thereby completing a cycle of heat transfer. The testing instruments are used to measure the flow rates, temperatures, pressures and pressure drops of cold and hot medium. The flow rate of liquid can be measured by contact-type flow rate sensor. The volume flow rate of vapour is measured by steam flowmeter or condensate weighing method. The temperature can be measured by temperature sensor which is placed in the center of the tube. The pressure and pressure drop can be measured by pressure transmitter or pressure difference transmitter. The accuracy levels and ranges of instruments should also meet the requirements.

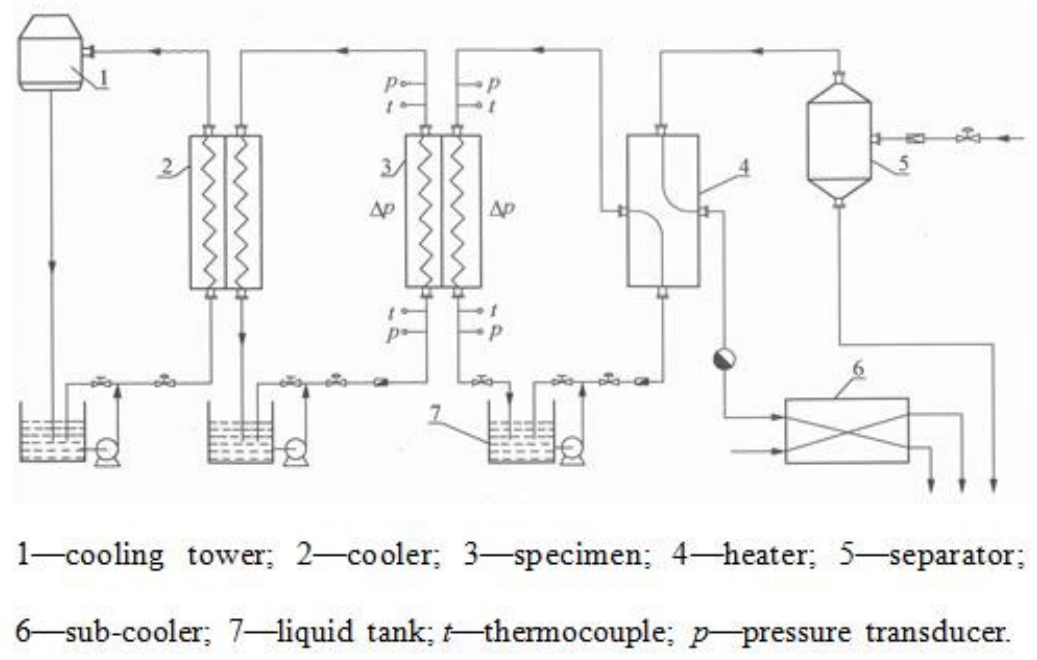

Figure 1. Testing system for convective heat transfer

\section{Case study}

\subsection{Testing condition}

The testing heat exchanger is an equal channel plate heat exchanger and both the cold and hot working medium is water. According to requirement of GB/T 27698-2011, the test conditions are divided into two cases. In case 1, the velocity of cold water is equal to that of hot water, changing from $0.2 \mathrm{~m} / \mathrm{s}$ to $0.9 \mathrm{~m} / \mathrm{s}$ with an interval of
$0.1 \mathrm{~m} / \mathrm{s}$, which is applicable to equal velocity method. In case 2 , the velocity of hot water is fixed at $0.5 \mathrm{~m} / \mathrm{s}$ and the velocity of cold water is changed from $0.2 \mathrm{~m} / \mathrm{s}$ to $0.9 \mathrm{~m} / \mathrm{s}$ with an interval of $0.1 \mathrm{~m} / \mathrm{s}$, which is applicable to modified Wilson plot method.

Table 1. Technical parameters of the testing sample

\begin{tabular}{ccc}
\hline technical parameters & unit & value \\
\hline number of plate & $/$ & 33 \\
thickness of plate & $\mathrm{mm}$ & 0.50 \\
\hline
\end{tabular}




\begin{tabular}{ccc}
\hline $\begin{array}{c}\text { equivalent diameter } \\
\text { cross-sectional area of each } \\
\text { channel }\end{array}$ & $\mathrm{mm}$ & 6.58 \\
$\begin{array}{c}\text { total heat transfer area } \\
\text { the }\end{array}$ & $\mathrm{m}^{2}$ & 0.024480 \\
\hline
\end{tabular}

\subsection{Overall heat transfer performance}

Figure 2 depicts the variation trend of overall heat transfer coefficient with velocity of cold fluid. The heat transfer coefficients in both curves rise with the increase of velocity of cold fluid. This is because the increase of velocity can enhance heat transfer performance of fluid on one side. The increasing rate in case 2 is larger than that in case 1 , owing to the increasing of velocity on both sides. Meanwhile, the two curves intersect at one point, in which the velocity of cold and hot fluid in both cases is at $0.5 \mathrm{~m} / \mathrm{s}$. This shows that the stability and accuracy of the test system are very high.

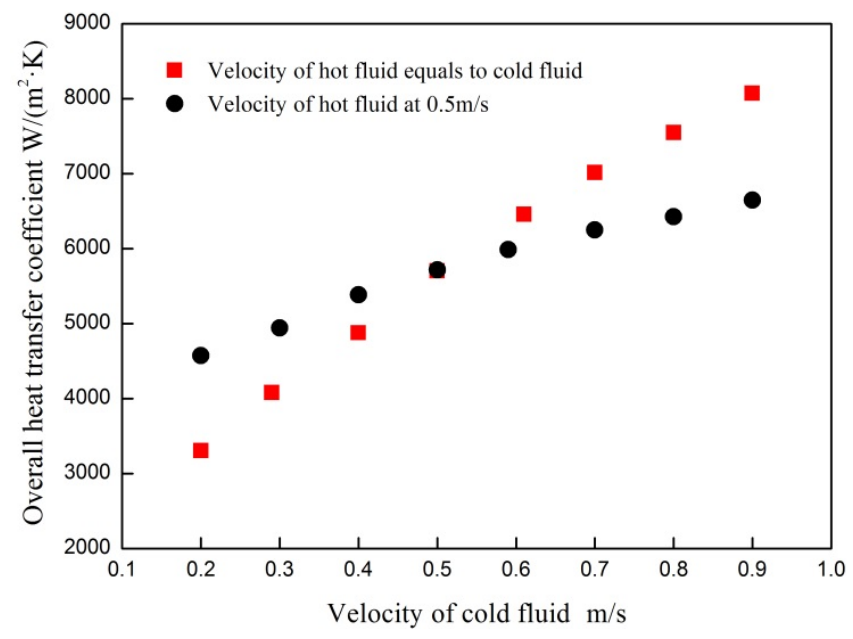

Figure 2. Variation trend of overall heat transfer coefficient with flow velocity

\subsection{Heat transfer correlations}

For case 1, the heat transfer correlations between $N u$ and $R e$ are obtained by equal velocity method, as shown in Eq. (8) and Eq. (9). For case 2, the heat transfer correlations between $\mathrm{Nu}$ and $\mathrm{Re}$ are obtained by modified Wilson plot method, as shown in Eq. (10) and Eq. (11). It should be noticed that iterative method is needed for solving undetermined coefficients. It is necessary to assume the initial value of the undetermined coefficients firstly. Finally the solved coefficients are compared with the initial value. The iterative process is ended until the error meets the requirements.

$$
N \mathrm{u}_{h}=0.2015 \cdot \operatorname{Re}_{h}^{0.7099} \operatorname{Pr}_{h}^{0.3} \quad\left(2200<\operatorname{Re}_{h}<11000\right)
$$

$$
\begin{aligned}
& N \mathrm{u}_{c}=0.2015 \cdot \operatorname{Re}_{c}^{0.7099} \operatorname{Pr}_{c}^{0.4} \quad\left(1800<\operatorname{Re}_{c}<8000\right) \\
& N \mathrm{u}_{h}=0.2008 \cdot \operatorname{Re}_{h}^{0.7142} \operatorname{Pr}_{h}^{0.3} \quad\left(2200<\operatorname{Re}_{h}<11000\right) \\
& N \mathrm{u}_{c}=0.2008 \cdot \operatorname{Re}_{c}^{0.7142} \operatorname{Pr}_{c}^{0.4} \quad\left(1800<\operatorname{Re}_{c}<8000\right)
\end{aligned}
$$

The $\mathrm{Nu} / \mathrm{Pr} 0.4$ obtained by equal velocity method is compared with that obtained by modified Wilson plot method, as shown in Fig. 3. It can be seen that the calculated $\mathrm{Nu} / \mathrm{Pr} 0.4$ according to the two correlations are basically equal. The values using modified Wilson plot method are slightly higher than that using equal velocity method, but the errors are all within $+5 \%$, as shown in Fig. 4. This shows that for plate heat exchangers with equal channel, the equal velocity method and modified Wilson plot method can both be used to test the convective heat transfer performance.

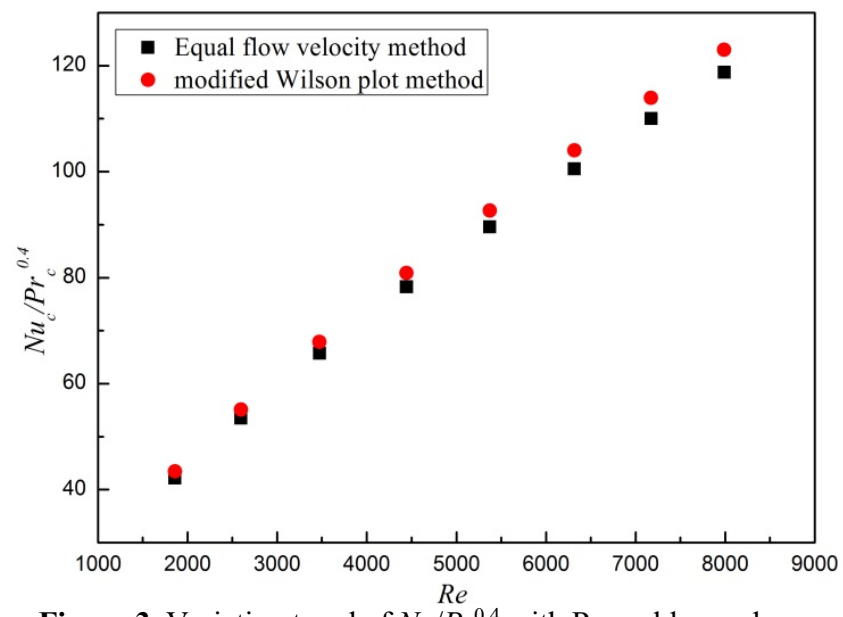

Figure 3. Variation trend of $\mathrm{Nu} / \mathrm{Pr}^{0.4}$ with Reynolds number 


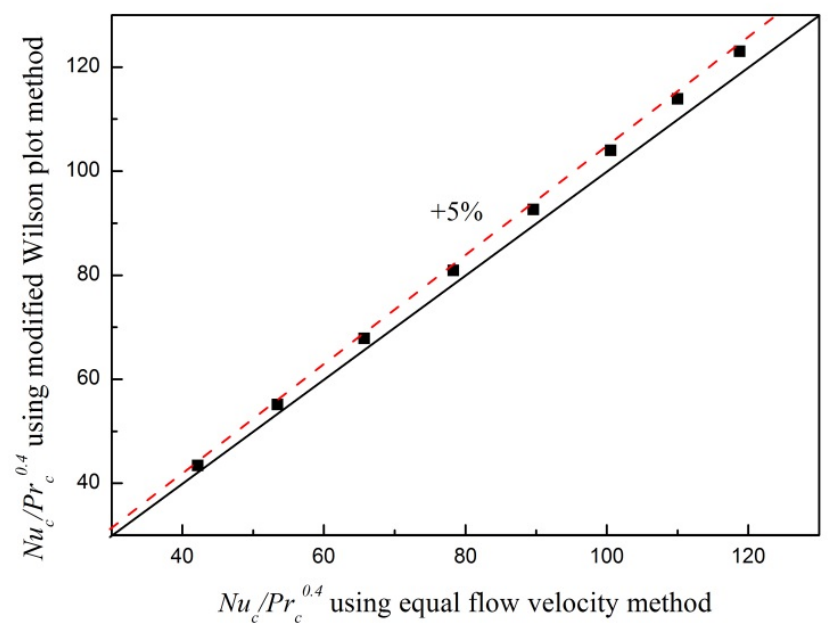

\section{Conclusions}

In this paper, the basic principles of modified Wilson plot method and equal velocity method are introduced. The testing system including flow chart and testing instruments are discussed. And the contrast experiments using the different two methods are conducted. The results showed that for plate heat exchangers with equal channel, the equal velocity method and modified Wilson plot method can both be used to test the convective heat transfer performance of plate heat exchanger. The equal velocity method is recommended because the deformation of plate is relatively smaller.

\section{Acknowledgments}

This work was funded by Shanghai Municipal Administration for Market Regulation (NO. 2019-22).

\section{References}

1. Lanzhou Petroleum Machinery Research Institute. (2013) Heat Exchanger (2nd Edition). China Petrochemical Press, Beijing.

2. Shi, M. Z., W, Z. Z. (2013) Principle and Design of Heat Exchangers (6th Edition). Southeast University Press, Nanjing.

3. National Boiler and Pressure Vessel Standardization Technical Committee. (2011) GB/T 27698 Test method for the performance of heat exchangers and heat exchange element (Chinese standards).

4. Ouyang, X. P., Wu, G. M., Liu, B. X. (2001) Equal Velocity Method and Its Application in Heat Transfer Performance Test of Plate Heat Exchangers. Power Engineering, 21(3): 12601262.

5. Zhang, H. Q., Tian, X. N., Jiang, Y. C. (2011) Comparison of Methods for Establishing Heat Transfer Rule Equation of Plate Heat Exchangers. Gas and Heat, 31(12): 56-59.
6. Wei, L. W., Li, S., Zhao, L. (2001) Heat transfer rule equation linear regression for heat exchangers. Petro-chemical Equipment, 34(3): 32-34. 